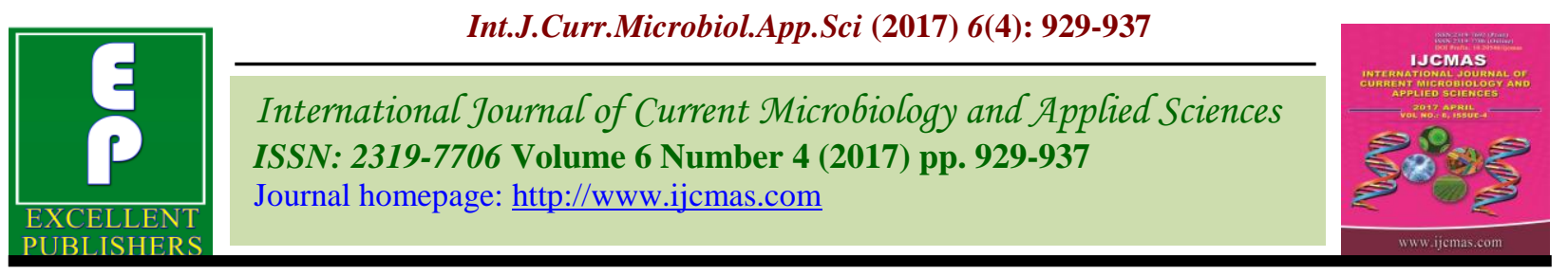

Original Research Article

https://doi.org/10.20546/ijcmas.2017.604.117

\title{
Haemophilus influenzae in Community Acquired Infections
}

\author{
Sai Keerthana* and Marina Thomas Appalaraju
}

Department of Microbiology, PSG IMS\&R, Coimbatore, India

*Corresponding author:

\begin{tabular}{|c|c|}
\hline & A B S T R A C T \\
\hline & \multirow{6}{*}{$\begin{array}{l}\text { Haemophilus influenzae is strictly a human pathogen, responsible for many diseases like } \\
\text { community acquired pneumonia, meningitis, sinusitis, epiglottitis and otitis media. } \\
\text { Haemophilus type b (Hib) was the most common strain responsible for all the infections. } \\
\text { Non type b strains were at an emerging risk. Ampicillin was commonly used but resistance } \\
\text { due to TEM-1gene is being reported. It is aimed to find out whether Hib continues to be } \\
\text { isolated from childhood cases of pneumonia and its susceptibility to the drugs of choice } \\
\text { and possible mechanisms of resistance. All purulent respiratory samples were processed } \\
\text { for isolation of Haemophilus influenzae. They were identified based on its X\&V factor } \\
\text { requirement and a negative Porphyrin synthesis test. Biotyping and Serotyping was done } \\
\text { using standard methods. Ampicillin resistance was characterized by phenotypic and } \\
\text { genotypic methods. From the results, out of } 1540 \text { purulent respiratory samples, } \\
\text { Haemophilus influenzae was isolated from } 103 \text { cases. Among the } 103 \text { isolates, } 69 \text { were } \\
\text { Nontype b and } 34 \text { were type b Haemophilus influenzae. All the Hib isolates were from } \\
\text { those above } 20 \text { years of age. Among } 15 \text { Ampicillin resistant isolates, } 13 \text { were positive for } \\
\text { TEM-1 gene. Finally, Nontype b isolates were predominant and Hib was isolated only } \\
\text { from adult age group. }\end{array}$} \\
\hline & \\
\hline $\begin{array}{l}\text { Hib, Non type } \\
\text { Porphyrin syntl } \\
\text { test, } \\
\text { X \&V factors, } \\
\text { TEM-1 }\end{array}$ & \\
\hline Article Info & \\
\hline $\begin{array}{l}\text { Acceptec } \\
\text { 06 Marc } \\
\text { Availabl } \\
10 \text { April }\end{array}$ & \\
\hline & \\
\hline
\end{tabular}

\section{Introduction}

Haemophilus influenzae is strictly a human pathogen responsible for many diseases like community acquired pneumonia, meningitis, sinusitis, epiglottitis and otitis media (Mac Neil et al., 2011). Haemophilus influenzae is a short pleomorphic Gram negative bacillary rod which is fastidious in nature. It is mainly recovered from the human respiratory tract and very rarely from other sites. The mode of spread of the infection is mainly airborne droplets (Puig et al., 2014). Among the 6 capsulated strains (a, b, c, d, e, f) type b remains the most common cause of invasive diseases (Puig et al., 2014; Jain et al., 2006). Type b H. influenzae colonizes the respiratory tract of children at a rate of 2- $4 \%$ and this has shown substantial decrease after the introduction of the conjugate vaccine. Non typeable strains account for $25-35 \%$ of otitis media in children less than 5 years of age and exacerbations of chronic obstructive pulmonary disease in adults (Urwin et al., 1996).

Haemophilus influenzae is confirmed by performing satellitism, in which the colony grows near a streak of Staphylococcus aureus. Haemophilus influenza is a fastidious organism which requires two important factors, namely, factor $\mathrm{X}$ (hemin factor) and factor $\mathrm{V}$ (Nicotinamide adenine nucleotide factor) for its growth. The detection of 
porphyrins produced from b-Aminolevulinic acid is evaluated for use in speciation of the Haemophilus genus (Lund et al., 1977). Porphyrin synthesis is positive for the non hemin requiring $H$. parainfluenzae and is negative for Haemophilus influenzae which requires both hemin and NAD factor for its growth.

Haemophilus influenzae can be classified in to eight biotypes based on three biochemical tests indole, Ornithine, urease and this has been used as an epidemiological marker for carriage in the upper respiratory tract. Of these biotypes, type $\mathrm{b}$ falls mostly in biotype I (Campos et al., 2004).

Infections caused by $H$. influenzae were initially being treated with cell wall active agents like Ampicillin and protein synthesis inhibitors like Chloramphenicol. Now the favoured regimen is Ceftrioxzone, Tetracycline and Azithromycin (Barbour et al., 1996; Guevara et al., 2008).Some of the Haemophilus influenzae shows resistance to ampicillin which is mainly due to beta lactamase production. Beta lactamase may be due to two genes, TEM-1 and ROB-1 genes, but the most commonly isolated gene is the TEM-1 gene. Both phenotypic and genotypic methods were used to detect Ampicillin resistance (CDC, 1998).

The aim of this study is to identify the serotype predominance in childhood and adult cases of pneumonia and to know the whether the vaccination against Hib has had any effect on the serotype predominance in different age groups and whether the isolates are susceptible to the drugs of choice like Ceftrioxzone, tetracycline and Azithromycin. The study also characterizes resistance to Ampicillin, which was used as an empirical treatment by the clinicians in earlier days for upper respiratory tract infections.

\section{Materials and Methods}

\section{Study population}

The study was done during the period from March 2014 to August 2015. A total of 1540 purulent respiratory samples from patients suspected of having community acquired pneumonia were processed in our microbiology laboratory.

\section{Isolation of Haemophilus influenzae}

All samples as indicated above were inoculated on Chocolate agar, Fildes agar, Chocolate agar with antibiotics (Vancomycin, Bacitracin and Clindamycin), and Blood agar with Staphylococcus streak.

\section{Blood agar (MacNeil et al., 2011)}

Blood agar was streaked with the sample along with Staphylococcus aureus and it was incubated at $37^{\circ} \mathrm{C}$ in the presence of $5-10 \%$ $\mathrm{CO}_{2}$ in a candle extinction jar for 24 hours. They form low, translucent, convex, pinpoint colonies around the staphylococcus which provides the factor $\mathrm{V}$ for its growth. The size of the colony decreases as it goes away from the staphylococcus streak. This is the satellite phenomenon shown by Haemophilus influenzae.

\section{Chocolate agar (Kilian, 1976)}

Heated blood agar or the Chocolate agar which provides both the hemin and NAD factor was streaked with the sample and it was incubated at $37^{0} \mathrm{C}$ in the presence of 5$10 \% \mathrm{Co}_{2}$ in a candle extinction jar for 24 hours. Haemophilus influenzae shows a shiny, tiny, smooth, flat colony which smells like wet fur. 
Fildes agar (Campos et al., 2004)

Fildes enrichment is an enzymatic digest of blood used to enhance the growth of fastidious organisms. The resultant digest is rich in growth factors including $\mathrm{X}$ and $\mathrm{V}$ factor required by Haemophilus influenzae. To the BHI chocolate agar of $100 \mathrm{ml}, 5 \mathrm{ml}$ of Fildes enrichment broth was added and allow the media to set. The sample is streaked and incubated at $37^{\circ} \mathrm{C}$ in the presence of $5-10 \%$ $\mathrm{CO}_{2}$ in a candle extinction jar for 24 hours. Colony morphology is very similar to that on chocolate agar.

\section{Chocolate agar with VBC (Harrison et al.,} 2009)

The CHOC-VBC media was made by preparing chocolate agar and the following antibiotics were added in concentrations of 5 microgram $/ \mathrm{ml} \quad$ Vancomycin, $\quad 300$ microgram $/ \mathrm{ml}$ of Bacitracin, 1 microgram $/ \mathrm{ml}$ of clindamycin 16.After that the media is allowed to dry and the samples are directly streaked on to the plates and incubated at $370 \mathrm{C}$ in the presence of $5-10 \% \mathrm{Co}_{2}$ in a candle extinction jar for 24 hours. Haemophilus influenzae shows a shiny, tiny, smooth, flat, slightly yellow colony which smells like wet fur.

Antibiotic susceptibility testing (Nag et al., 2001)

Antibiotic susceptibility testing was done on chocolate agar for susceptibility to Ceftrioxzone, Azithromycin, Aztreonam, ciprofloxacin, ampicillin, tetracycline, Cotrimoxazole and Meropenem based on 2014 CLSI guidelines. Ampicillin resistant strains were then tested for beta lactamase production by acidimetric method. One of the genes responsible for beta lactamase production (TEM-1) was identified by conventional PCR. The following primers are used for detection of TEM-1 gene:-
5'TGGGTGCACGAGTGGGTTAC3' and 5'TTATCCGCCTCCATCCAGTC3'

Porphyrin synthesis test (Campos et al., 2004)

This test indicates the absence of requirement of $\mathrm{X}$ factor. If $\alpha$-Amino levulinic acid is provided to the bacterium, it synthesizes it and excretes porphobilinogen and other porphyrins. This test is carried out by using kovac's indole reagent (Para dimethyl amino Benzaldehyde) and formation of pinkish red colour in the lower water phase indicates the synthesis of porphyrin and absence of $\mathrm{X}$ factor requirement.

\section{$\mathrm{X}$ and V requirement (Madore, 1996)}

Make a lawn culture in the Muller Hinton agar plate and keep the commercially available discs $\mathrm{X}$ and $\mathrm{V}$ from HIMEDIA laboratories. The $\mathrm{X}$ factor (hemin) and $\mathrm{V}$ factor (Coenzyme- Nicotinamide adenine dinucleotide NAD+) are impregnated on the sterile filter paper discs of diameter $6 \mathrm{~mm}$. If both $\mathrm{X}$ and $\mathrm{V}$ factors are

\section{Typing}

i) Biotyping was done using the biochemical tests as follows: - Indole production, Ornithine decarboxylation and urease production (Campos et al., 2004).

ii) Serotyping was done using -BectonDickinson --- Kit.

iii)BD Difco catalogue no. Type b-222361

iv) Type a-222501

v) Type f-227921

\section{Results and Discussion}

Among the 1540 cases of suspected pneumonia, the purulent respiratory samples yielded. Haemophilus influenzae were noted in 103 cases. Out of 103 isolates, 84 were 
from inpatient samples and 19 were from outpatient samples. Among 84 inpatient cases, $33 \%$ were associated with COPD, $26.2 \%$ with Bronchial asthma and $18.44 \%$ were provisionally diagnosed as pyrexia of unknown origin.

\section{Distribution of Haemophilus influenzae in various age groups}

Among the 103 Haemophilus influenzae isolates, $95(92 \%)$ of them were isolated from patients above 20 years of age and $8(7.7 \%)$ from below 20 years. Among the 95 strains of Haemophilus influenzae isolated from adults, $63(61.2 \%)$ were in males and 40(38.8\%) in female patients. The strains isolated were more during winter months of October to January (Figure 1).

\section{Serotype}

All the isolates of Haemophilus influenzae were serotyped with type $\mathrm{b}$, type a and type $\mathrm{f}$ antiserum. A total of 34(33\%) were type b and the remaining 69 were non-type b. Among the 69 non type b strains, $46.6 \%$ were other serotypes, $10.7 \%$ were type a and $9.7 \%$ were type f. (Figure 2). Comparison of Haemophilus influenzae serotype $\mathrm{b}$ in different age groups showed that all isolates of type b were from those above 20 years, whereas no type $b$ were isolated from below 20 years and this is statistically significant $(\mathrm{p}<$ $0.05)$.

\section{Biotypes}

Haemophilus influenzae can be divided in to eight biotypes based on three biochemical tests, namely, Indole, Ornithine and Urease production. Out of the 103 Haemophilus influenzae isolates, strains belonging to all 8 biotypes were detected and 47 strains belonged to biotype I (Table 1).

\section{Antibiotic sensitivity pattern}

Antibiotic sensitivity testing of 103 isolates of Haemophilus influenzae showed that $91.2 \%$ were sensitive to Ceftrioxzone, $96.1 \%$ to Azithromycin, 99.02\% to Aztreonam, 94.2\% to ciprofloxacin, $85.4 \%$ to Ampicillin, $96.1 \%$ to Meropenem, $97.08 \%$ to Tetracycline and $65.04 \%$ to Cotrimoxazole.

\section{Detection of Ampicillin resistance}

Among the 103 isolates of Haemophilus influenzae isolated, 15(14.6\%) strains showed Ampicillin resistance. Out of the 15 Ampicillin resistant strains, all 15 produced beta lactamases by acidimetric method and 13 showed presence of TEM-1 (Figure 3).

Haemophilus influenzae causes a variety of community acquired respiratory infections, including sinusitis, acute otitis media, bronchitis and community acquired pneumonia. Earlier, these infections were mainly caused by Haemophilus influenzae serotype b (Hib), but after the introduction of vaccines, the incidence of Haemophilus influenzae type b has markedly reduced.In our study, Non type b Haemophilus influenzae has emerged as an increasing cause of the pneumonia $(67 \%)$ as compared to type $b$ and this is similar to other studies also where it is isolated in 70-90\% cases (Mac Neil et al., 2011; Puig et al., 2014) and Jain et al., (2006).

Earlier studies done during the period from 1993-1997, show a high rate (86\%) of isolation of Hib amongst children below 5 years of age (Lund et al., 1977). Studies between 1996 and 2004 have reported lower isolations of 26-28\% among children (Urwin et al., 1996; Campos et al., 2004 and Barbour (1996). 
Among children in Asia and central European countries a considerable decrease has been demonstrated from 21 cases per 1, 00,000 populations per year in 1994 to 5 cases per 1 , 00,000 populations per year by 2013 (MacNeil et al., 2011; Puig et al., 2014). A comparative study in Costa Rica have also reported a significant decline in the incidence of type b $H$. influenzae when the data from 1992 -1997 was compared with that between 1999 and 2004 (Guevara et al., 2008). By June 2011, Hib vaccine was included in the National Immunization program of 170 countries in all regions of the world. After that the incidence of Hib invasive disease and oropharyngeal carriage in young children has drastically decreased to $0.3-0.7$ cases per 1 , 00,000 populations per year wherever vaccination programs have been implemented (Campos et al., 2004; Barbour, 1996 and Madore, 1996). In our study none of the isolates were from cases below 20 years of age.

The $H$. influenzae isolates in this study were more commonly seen among male patients (61\%), which are similar to other studies (Urwin et al., 1996). In contrast, a study reports higher prevalence among female elderly patients, and a lower prevalence in female children (Mac Neil et al., 2011). This infection has always shown a seasonal variation. Isolation of $H$. influenzae has shown higher incidence during winter months across many countries (Kilian, 1976; Zhu et al., 2015). In our study also $61 \%$ were isolated during winter months. An increase in isolations during rainy season is also observed in some studies (Guevara et al., 2008; Zhu et al., 2015 and Leibovitz et al., 2004).

In this study, strains belonging to all known biotypes were isolated and $44 \%$ were Biotype I whereas in other studies $60-70 \%$ belonged to this biotype (Landgraf et al., 1993). COPD was an important risk factor for the development of Haemophilus influenzae pneumonia and their association was 25 to $30 \%$ percent in many studies (Jain et al., 2006; Urwin et al., 1996), when compared to $33 \%$ in our study. Association of Haemophilus influenzae with bronchial asthma cases were $26.2 \%$ in our study which correlated well with many studies where $28 \%$ association is noted (Jain et al., 2006; Urwin et al., 1996).

Ceftrioxzone being the drug of choice for invasive $H$. influenzae infections, $91 \%$ of our isolates were susceptible, and this correlated well (95\%-97\%) with other studies in both developed and developing countries (Zhu et al., 2015; Harrison et al., 2009). Infections caused by Haemophilus influenzae, particularly sinusitis and otitis media are now frequently treated with fluroquinolones and their sensitivity were $94 \%$ in our study which is comparable to other studies (Farrell et al., 2005).

A susceptibility of $94.5 \%$ in 2012 as compared to $54 \%$ in the year 2000 was observed with tetracycline and this remarkable increase in susceptibility is reportedly due to decrease in its usage in the past 20 years (Bennett et al., 2014). In this study also $97 \%$ of isolates were sensitive to tetracycline.

Azithromycin is an acid stable orally administered Macrolides antimicrobial drug that appears to be more active against many Gram-positive pathogens and several other Gram negative pathogens, notably Haemophilus influenzae. After oral administration, serum concentrations of Azithromycin are lower reflecting the rapid and extensive movements of the drug from the circulation into intracellular compartments resulting in increased tissue concentrations (Puig et al., 2015). In our study 99\% were susceptible to Azithromycin, which was similar to other studies (Appelbaum et al., 2003; Jacobs et al., 1999; Doern et al., 1997). 
High level resistances to Macrolides in $H$. influenzae $(0.5 \%-2 \%)$ are rare as is reported in many studies (Appelbaum et al., 2003; Jacobs et al., 1999; Doern et al., 1997). Macrolides are of particular use in children who are allergic to beta lactams (Jacobs et al.,
1999). There was no difference in susceptibility between type $b$ and non-type $b$ isolates to Ceftrioxzone, tetracycline, Azithromycin and ciprofloxacin (Zhu et al., 2015; Nag et al., 2001).

Table.1 Biotype distribution among different isolates of Haemophilus influenza

\begin{tabular}{|c|c|c|}
\hline Biotypes & $\begin{array}{l}\text { Number of isolates showing } \\
\text { different biotypes }\end{array}$ & Percentage \\
\hline I & 47 & $45 \%$ \\
\hline II & 6 & $6 \%$ \\
\hline III & 5 & $5 \%$ \\
\hline IV & 1 & $1 \%$ \\
\hline V & 2 & $2 \%$ \\
\hline VI & 5 & $5 \%$ \\
\hline VII & 4 & $4 \%$ \\
\hline VIII & 33 & $32 \%$ \\
\hline
\end{tabular}

Haemophilus influenzae isolates, strains belonging to all 8 biotypes were detected and 47 strains belonged to biotype I (Table 1).

Fig.1 Seasonal variation of Haemophilus influenzae

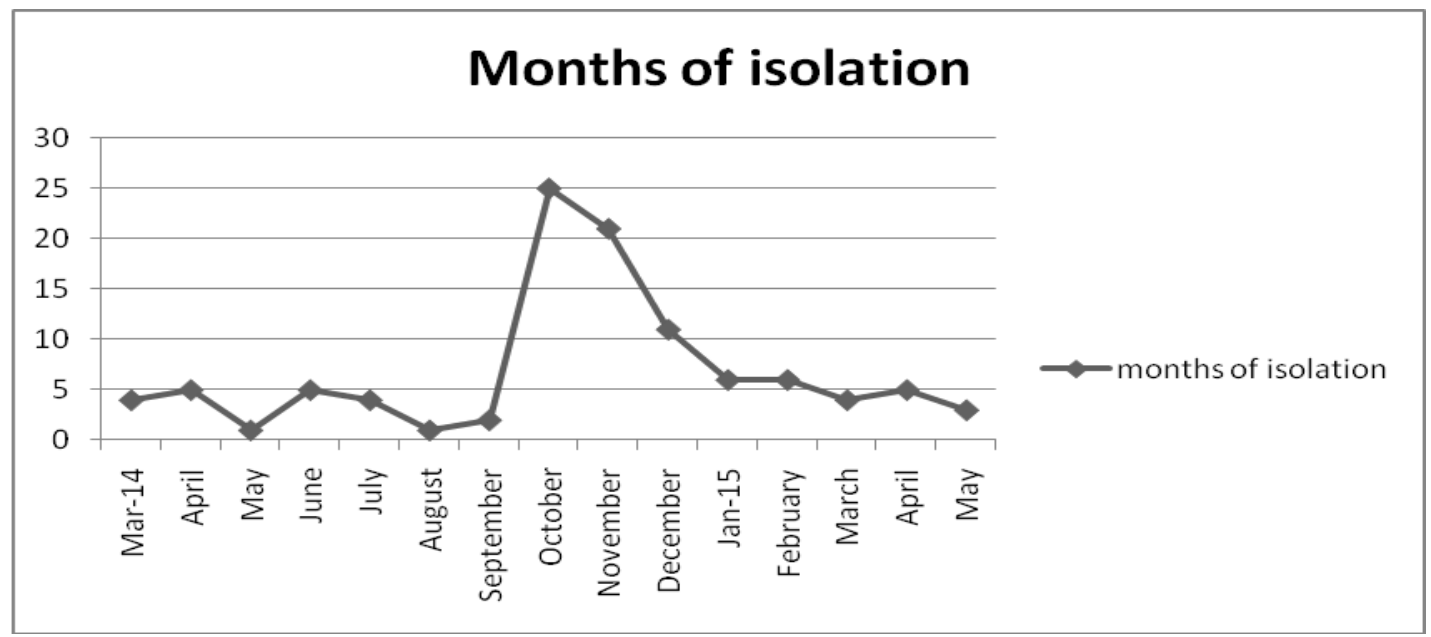

The strains isolated were more during winter months of October to January 
Fig.2 Serotypes in different age groups

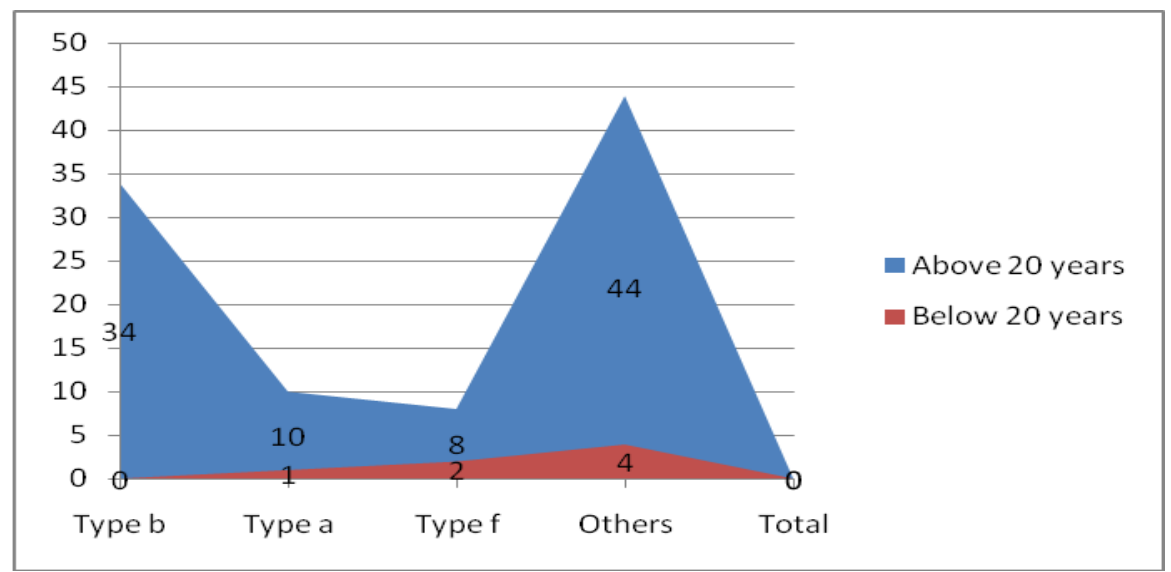

No type b was isolated from below 20 years and this is statistically significant $(\mathrm{p}<0.05)$

Fig.3 TEM-1 gene detection by PCR

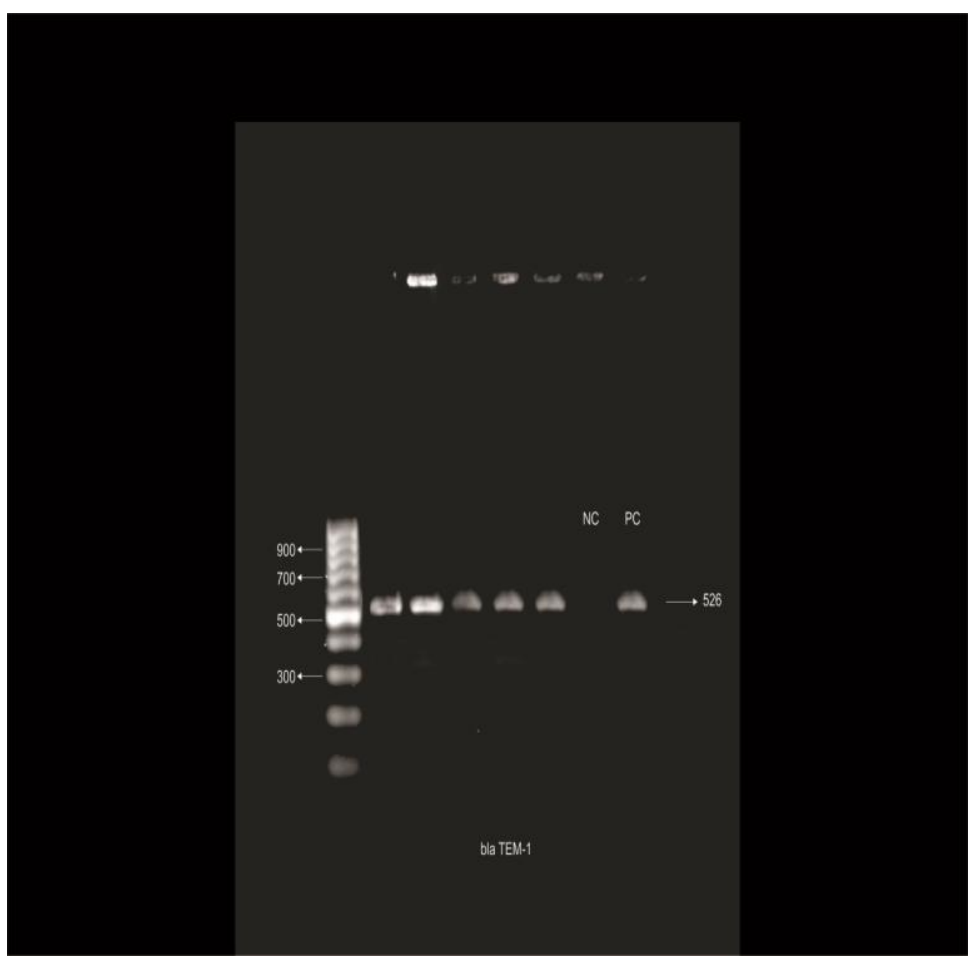

Beta lactamase mediated resistance was seen in $14.6 \%$ isolates in this study, whereas in multicentre studies done in US between 19841999 it was $15 \%$ to $36 \%$ (Nag et al., 2001) and in recent studies done in China, the percentage of Ampicillin resistant strains progressively increased from $4 \%$ in the year 2000 to $29 \%$ in 2012 (Zhu et al., 2015). The resistance detected was higher among type $b$
(60\%) strains when compared to non type b strains $(40 \%)$ which is similar to study by Nag et al., (30\% and 20\%) suggesting that the strains that are invasive is showing a higher ampicillin resistance (Nag et al., 2001).

All the ampicillin resistant isolates produced beta-lactamase enzyme when screened by the phenotypic acidimetric method, but when 
analyzed by molecular method, only 13 isolates (86\%) were positive for TEM-1 gene which is similar to many studies where the detection of TEM-1 gene (93\%) is more when compared to the ROB-1gene (5\%) detection (Hasegawa et al., 2003; Farrell et al., 2005 and Kuvat et al., 2015).

\section{Acknowledgements}

I thank Dr. S. Ramalingam and Dr. B. Appalaraju for permitting me to carry out the work in the Department of Microbiology, PSG IMS\&R, Coimbatore. I acknowledge the kind help rendered by the supervisor Dr. Marina Thomas for having guided at every level.

\section{References}

Appelbaum, P.C., Peric, M., Bozdogan, B., Jacobs, M.R. 2003. 'Effects of an Efflux Mechanism and Ribosomal Mutations on Macrolides Susceptibility of Haemophilus influenzae Clinical Isolates'. Antimicrobial Agents Chemother., p. 1017-1022.

Barbour, M.L. 1996. 'Conjugate vaccines and the carriage of Haemophilus influenzae type b'. Emerg Infect. Dis., 2(3): 176.

Bennett, J.E., Dolin, R., Blaser, M.J. 2014. 'Principles and practice of infectious diseases'. Elsevier Health Sci.

Campos, J., Hernando, M., Roman, F., Perez Vazquez, M., Aracil, B., Oteo, J., Lazaro, E., de Abajo, F. 2004. Analysis of invasive Haemophilus influenzae infections after extensive vaccination against $H$. influenzae type b'. J Clin. Microbiol., 42(2): 524-9.

Centers for Disease Control and Prevention. 1998. 'Progress toward eliminating Haemophilus influenzae type $\mathrm{b}$ disease among infants and children'--United States, 1987-1997. MMWR. Morbidity and Mortality Weekly Report, 47(46): 993.

Doern, G.V., Brueggemann, A.B., Pierce, G.,
Holley, H.P., Rauch, A. 1997. 'Antibiotic resistance among clinical isolates of Haemophilus influenzae in the United States in 1994 and 1995 and detection of beta-lactamase-positive strains resistant to amoxicillin-Clavulanate: results of a national multicenter surveillance study'. Antimicrobial Agents and Chemother., 41(2): 292-7.

Farrell, D.J., Morrissey, I., Bakker, S., Buck ridge, S., Felmingham, D. 2005. 'Global distribution of TEM-1 and ROB-1 $\beta$ lactamases in Haemophilus influenzae'. J. Antimicrobial Chemother., 56(4): 773-6.

Guevara, S., Soley, C., Arguedas, A., Porat, N., Dagan, R. 2008. 'Seasonal distribution of Otitis media pathogens among Costa Rican children'. The Pediatric Infect. Dis. J., 27(1): 12-6.

Hasegawa, K., Yamamoto, K., Chiba, N., Kobayashi, R., Nagai, K., Jacobs, M.R., Appelbaum, P.C., Sunakawa, K., Ubukata, K. 2003. 'Diversity of Ampicillin-resistance genes in Haemophilus influenzae in Japan and the United States'. Microbial Drug Resistance, 9(1): 39-46.

Harrison, C.J., Woods, C., Stout, G., Martin, B., Selvarangan, R. 2009. 'Susceptibilities of Haemophilus influenzae, Streptococcus pneumoniae, including serotype 19A, and Moraxella catarrhalis paediatric isolates from 2005 to 2007 to commonly used antibiotics'. J. Antimicrobial Chemother.

Jacobs, M.R., Bajaksouzian, S., Zilles, A., Lin, G., Pankuch, G.A., Appelbaum, P.C. 1999. 'Susceptibilities of Streptococcus pneumoniae and Haemophilus influenzae to 10 oral antimicrobial agents based on pharmacodynamic parameters: 1997 US surveillance study'. Antimicrobial Agents and Chemother., 43(8): 1901-8.

Jain, A., Kumar, P., Awasthi, S. 2006. 'High Ampicillin resistance in different biotypes and serotypes of Haemophilus influenzae colonizing the nasopharynx of healthy school-going Indian children'. J. Med. Microbiol., 55(2): 133-7.

Kilian, M. 1976. 'A taxonomic study of the 
genus Haemophilus, with the proposal of a new species'. J. Clin. Microbiol., 93(1): 9-62.

Kuvat, N., Nazik, H., Berkiten, R., Ongen, B. 2015. 'TEM-1 and ROB-1 presence and Antimicrobial Resistance in Haemophilus influenzae Strains, Istanbul, Turkey'. The Southeast Asian J. Trop. Med. Public Health, 46(2): 254-61.

Landgraf, I.M., Vieira, M.F. 1993. 'Biotypes and serotypes of Haemophilus influenzae from patients with meningitis in the city of Sao Paulo, Brazil'. J. Clin. Microbiol., 31(3): 743-5.

Leibovitz, E., Jacobs, M.R., and Dagan, R. 'Haemophilus influenzae: a significant pathogen in acute Otitis media'. The Pediatric Infect. Dis. J., 23(12): 1142-52.

Lund, M.E., Blazevic, D.J. 1977. Rapid speciation of Haemophilus with the porphyrin production test versus the satellite test for X.' J. Clin. Microbiol., 5(2): 142-144.

Mac Neil, J.R., Cohn, A.C., Farley, M., Mair, R., Baumbach, J., Bennett, N., Gershman, K., Harrison, L.H., Lynfield, R., Petit, S., Reingold, A. 2011. 'Current epidemiology and trends in invasive Haemophilus influenzae disease'-United States, 1989-2008. Clin. Infect. Dis., 53(12): 1230-1236.

Madore, D.V. 1996. 'Impact of immunization on Haemophilus influenzae type b disease'. Infectious Agents and Dis., 5(1): 8-20.
Nag, V.L., Ayyagari, A., Venkatesh, V., Ghar, M., Yadav, V., Prasad, K.N. 2001. 'Drug resistant Haemophilus influenzae from respiratory tract infection in a tertiary care hospital in north India'. Indian J. Chest Dis. Allied Sci., 43(1): 13-8.

Puig, C., Grau, I., Marti, S., Tubau, F., Calatayud, L., Pallares, R., Liñares, J., Ardanuy, C. 2014. 'Clinical and molecular epidemiology of Haemophilus influenzae causing invasive disease in adult patients'. PLoS one, 9(11): e112711.

Puig, C., Tirado-Velez, J.M., Calatayud, L., Tubau, F., Garmendia, J., Ardanuy, C., Marti, S., Adela, G., Liñares, J. 2015. 'Molecular characterization of fluroquinolones resistance in nontypeable Haemophilus influenzae clinical isolates'. Antimicrobial Agents and Chemother., 59(1): 461-466.

Urwin, G., Krohn, J.A., Robinson, K.D., Wenger, J.D., Farley, M.M. 1996. Haemophilus influenzae Study Group.' Invasive disease due to Haemophilus influenzae serotype $\mathrm{f}$ : clinical and epidemiologic characteristics in the $H$. influenzae serotype b vaccine era'. Clin. Infect. Dis., 22(6): 1069-76.

Zhu, H., Wang, A., Tong, J., Yuan, L., Gao, W., Shi, W., Yu, S., Yao, K., Yang, Y. 2015. 'Nasopharyngeal carriage and antimicrobial susceptibility of Haemophilus influenzae among children younger than 5 years of age in Beijing, China'. BMC Microbiol., 15(1): 1.

\section{How to cite this article:}

Sai Keerthana, Marina Thomas Appalaraju. 2017. Haemophilus influenzae in Community Acquired Infections. Int.J.Curr.Microbiol.App.Sci. 6(4): 929-937. doi: https://doi.org/10.20546/ijcmas.2017.604.117 